\title{
Arsenic trioxide synergizes with everolimus (Rad001) to induce cytotoxicity of ovarian cancer cells through increased autophagy and apoptosis
}

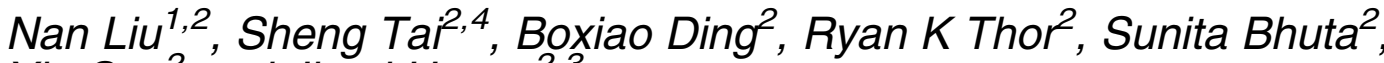 \\ Yin Sun and Jiaoti Huang $^{2,3}$
}

\begin{abstract}
${ }^{1}$ Department of Obstetrics and Gynecology, Nanfang Hospital, Southern Medical University, 1838 North Guangzhou Avenue, Guangzhou, Guangdong 510515, People's Republic of China

${ }^{2}$ Department of Pathology and Laboratory Medicine, David Geffen School of Medicine at the University of California at Los Angeles, 10833 Le Conte Avenue, 13-229 CHS, Los Angeles, California 90095-1732, USA

${ }^{3}$ Jonsson Comprehensive Cancer Center and Broad Center for Regenerative Medicine and Stem Cell Biology, David Geffen School of Medicine at the University of California at Los Angeles, Los Angeles, California, USA

${ }^{4}$ Department of Urology and Anhui Geriatric Institute, The First Affiliated Hospital of Anhui Medical University, Hefei, Anhui, People's Republic of China
\end{abstract}

(Correspondence should be addressed to N Liu at Department of Obstetrics and Gynecology, Nanfang Hospital, Southern Medical University; Email: nanobgyn1225@gmail.com; J Huang at Department of Pathology and Laboratory Medicine, David Geffen School of Medicine at the University of California at Los Angeles; Email: jiaotihuang @ mednet.ucla.edu)

\begin{abstract}
Phosphatidylinositol 3-kinase/AKT/mammalian target of rapamycin pathway plays a key role in the tumorigenesis of a variety of human cancers including ovarian cancer. However, inhibitors of this pathway such as Rad001 have not shown therapeutic efficacy as a single agent for this cancer. Arsenic trioxide (ATO) induces an autophagic pathway in ovarian carcinoma cells. We found that ATO can synergize with Rad001 to induce cytotoxicity of ovarian cancer cells. Moreover, we identified synergistic induction of autophagy and apoptosis as the likely underlying mechanism that is responsible for the enhanced cytotoxicity. The enhanced cytotoxicity is accompanied by decreased p-AKT levels as well as upregulation of ATG5-ATG12 conjugate and LC3-2, hallmarks of autophagy. Rad001 and ATO can also synergistically inhibit tumors in a xenograft animal model of ovarian cancer. These results thus identify and validate a novel mechanism to enhance and expand the existing targeted therapeutic agent to treat human ovarian cancer.
\end{abstract}

Endocrine-Related Cancer (2012) 19 711-723

\section{Introduction}

Ovarian cancer is the fifth most common cancer in women and the leading cause of death among gynecological malignancies. It was reported that 21880 women were diagnosed with ovarian cancer and 13850 died from this disease in 2010 in the United States alone, and the overall 5-year survival rate from 1999 to 2006 was 45.6\% (Sfakianos \& Havrilesky 2011). The standard systemic treatment for advanced ovarian cancer is combination chemotherapy, using platinum-based drugs such as cisplatin coupled with paclitaxel. Because conventional chemotherapeutic agents target rapidly dividing cells including normal white blood cells, gastrointestinal epithelial cells, and hair follicle cells as well as cancer cells, significant side effects are common. In addition, chemoresistance often occurs, leading to death of the patients (Nick et al. 2011). Therefore, there is an urgent need to develop targeted systemic therapies.

The phosphatidylinositol 3-kinase (PI3K)-AKTmammalian target of rapamycin (mTOR) pathway is a major signaling pathway promoting cellular proliferation in many cancer types including ovarian cancer (Bartholomeusz \& Gonzalez-Angulo 2012). Rapamycin or its derivative, everolimus (we will refer to Rad001, which is a more stable derivative, hereafter), 
has shown therapeutic effect in certain cancers such as advanced kidney tumor and neuroendocrine pancreatic tumor. Rad001 inhibits the proliferation of ovarian cancer cell growth in vitro and in vivo (Mabuchi et al. 2011). Unfortunately, despite some reported benefits, the clinical results of Rad001 as a single agent have been less than encouraging (Guertin \& Sabatini 2007, Liu et al. 2009). A likely explanation for the limited efficacy of Rad001 is that inhibition of mTOR leads to compensatory activation of other signaling molecules, leading to continued proliferation of the cancer cells (Liu et al. 2009). Accumulating evidence suggests that combinations of Rad001 with chemotherapeutics such as paclitaxel and cisplatin can induce a stronger apoptotic response and enhanced antitumor effects compared with single-agent therapy (Mondesire et al. 2004, Campone et al. 2009). Because combination therapies have the potential to inhibit multiple pathways simultaneously, it is more likely to achieve superior therapeutic efficacy and decreased side effects (Mazzoletti et al. 2011).

Arsenic trioxide (ATO) is an effective chemotherapeutic drug for the treatment of acute promyelocytic leukemia (APL; Qian et al. 2007, Zhang et al. 2009, Chiu et al. 2011). The remarkable efficacy of ATO in the treatment of APL has led to much interest in its anticancer activity and underlying mechanism in other malignancies. Here, we report the synergistic anticancer effect of ATO with Rad001 and the underlying mechanisms in human ovarian cancer cells in vitro and in vivo. We found that the enhanced cytotoxic effect of Rad001 combined with ATO was through enhanced induction of autophagy and apoptosis. In addition, we found that inhibition of PI3K/AKT signaling pathway is likely the underlying mechanism for induced autophagy and apoptosis. These findings suggest a potentially more efficacious therapeutic strategy for ovarian cancer.

\section{Materials and methods}

\section{Materials}

The SKOV3 and OV2008 cell lines were kindly provided by Dr Dorigo of Department of Obstetrics and Gynecology at UCLA. Both cell lines used in this study were passaged for fewer than 6 months after resuscitation. Fetal bovine serum (FBS), RPMI 1640 medium, DMEM, sodium pyruvate, L-glutamine, penicillin, and streptomycin were purchased from Hyclone (Logan, UT, USA); ATO and Rad001 were from Sigma; CellTiter Glo assay was from Promega; Caspase-3/7 Assay Kit was from Anaspec
(Fremont, CA, USA); Annexin V apoptosis detection Kit was from eBioscience (San Diego, CA, USA); Lipofectamine 2000 transfection reagent was from Invitrogen (New York, NY, USA); rabbit anti-LC-3 polyclonal antibody was from GenScript (Piscataway, NJ, USA); rabbit anti-Beclin1, rabbit anti-cleaved caspase-3, and rabbit anti-PARP1 polyclonal antibodies, antibodies recognizing p70S6K, phosphop70S6K (Thr389), AKT, phospho-AKT (Ser473), 4E-BP1, p-4E-BP1 (Thr37/46), eIF-4E, p-eIF-4E (Ser209), c-Jun- $\mathrm{NH}_{2}$-terminal kinase (JNK), and p-JNK were from Cell Signaling (Danvers, MA, USA); rabbit anti-Atg5 monoclonal antibody was from Epitomics (Burlingame, CA, USA); rabbit polyclonal antibody to SQSTM1/p62 (sequestosome 1) was from GeneTeX (Irvine, CA, USA); and mouse anti- $\beta$-actin antibody was from Sigma.

\section{Cell culture}

SKOV3 cells were maintained in DMEM containing $10 \%$ FBS, penicillin-streptomycin, L-glutamine, $\mathrm{Na}^{+}$ pyruvate, and OV2008 cells were maintained in RPMI 1640 supplemented with $10 \%$ FBS and penicillinstreptomycin. Cells were grown at $37{ }^{\circ} \mathrm{C}$ with $5 \% \mathrm{CO}_{2}$.

\section{Cell relative luminescence units, viability, and combination index}

The cells were seeded into the 96-well plate at $3 \times 10^{3}$ cells/well. After $48 \mathrm{~h}$, SKOV3 cells were treated with DMSO, 0.047-0.746 $\mu \mathrm{M}$ Rad001, 1.399-22.380 $\mu \mathrm{M}$ ATO, or a combination of the two for $18 \mathrm{~h}$. OV2008 cells were treated with DMSO, $0.180-2.80 \mu \mathrm{M}$ Rad001, 6.00-98.00 $\mu \mathrm{M}$ ATO, or a combination of the two for $18 \mathrm{~h}$. Cells were plated in triplicates for each treatment group. Relative cell luminescence units were obtained using the Promega CellTiter Glo assay according to manufacturer's instructions. The compound interactions were analyzed with CalcuSyn software (version 2.1, Biosoft, Cambridge, UK) to determine the combination index (CI) for the combinatorial treatment of Rad001 and ATO. The SKOV3 and OV2008 cells were also treated with compounds for $18 \mathrm{~h}$ and analyzed with Trypan Blue staining.

\section{GFP-LC3 analysis}

The cells were transfected with GFP-LC3 plasmid using Lipofectamine 2000 transfection reagent. After $24 \mathrm{~h}$, the medium was changed, and the SKOV3 cells were treated with DMSO (control), $\operatorname{Rad} 001(0.37 \mu \mathrm{M})$, ATO $(11.19 \mu \mathrm{M})$, or a combination 
of $\operatorname{Rad001}(0.37 \mu \mathrm{M})$ and ATO $(11.19 \mu \mathrm{M})$ respectively for $18 \mathrm{~h}$. The OV2008 cell lines were treated with DMSO (control), Rad001 $(0.35 \mu \mathrm{M})$, ATO $(12.00 \mu \mathrm{M})$, or a combination of $\operatorname{Rad} 001(0.35 \mu \mathrm{M})$ and ATO $(12.00 \mu \mathrm{M})$ respectively for $18 \mathrm{~h}$. The cells were then fixed in $4 \%$ paraformaldehyde for $30 \mathrm{~min}$. The cells were washed twice with PBS and stained with DAPI and observed under a fluorescence microscope (Eclipse 90i slide scope) with $40 \times$ lense.

\section{Protein analysis}

The cultured cells were washed with cold PBS and lysed with lysis buffer $(20 \mathrm{mM} \mathrm{KCl}, 150 \mathrm{mM} \mathrm{NaCl}$, $1 \% \mathrm{NP}-40,50 \mathrm{mM} \mathrm{NaF}, 50 \mathrm{mM}$ Tris- $\mathrm{HCl}$, pH 7.5, $1 \mathrm{mM}$ dithiothreitol, $1 \mathrm{mM}$ EGTA, $1 \times$ protease inhibitor, $10 \%$ glycerol) for $30 \mathrm{~min}$ on ice. The cells were centrifuged for $20 \mathrm{~min}$ at $4{ }^{\circ} \mathrm{C}$. The protein concentration in the supernatant was determined with the Bradford assay (Bio-Rad). Equal amount of protein was loaded on 8 or $15 \%$ SDS-polyacrylamide gels and transferred to polyvinylidene fluoride membrane. The membrane was blocked with nonfat dry milk for $1 \mathrm{~h}$, incubated with primary antibody in nonfat dry milk overnight, washed with PBS/0.1\% Tween 20 for $30 \mathrm{~min}$, incubated with secondary antibody for $1 \mathrm{~h}$, washed with PBS/ $0.1 \%$ Tween 20 for $1 \mathrm{~h}$, and detected with enhanced chemiluminescence (Pierce, Rockford, IL, USA).

\section{Caspase-3/7 activity analysis}

Equal numbers of SKOV3 and OV2008 cells were seeded into the 96-well plates. After 24 h, SKOV3 cells were treated with DMSO (control), Rad001 $(0.37 \mu \mathrm{M})$, ATO $(11.19 \mu \mathrm{M})$, or the combination respectively for 18 h. OV2008 cell were treated with DMSO (control), $\operatorname{Rad} 001(0.35 \mu \mathrm{M})$, ATO $(12.00 \mu \mathrm{M})$, or the combination respectively for $18 \mathrm{~h}$. The caspase-3/7 activity was measured by the SensoLyte Homogeneous AMC Caspase-3/7 Assay kit after reaction for $1 \mathrm{~h}$. All the experiments were carried out in triplicates.

\section{Analysis of apoptosis}

After $18 \mathrm{~h}$ treatment, the SKOV3 and OV2008 cells were collected. The apoptosis was quantified by Flow cytometry with Annexin-V 7-AAD apoptosis detection Kit following the manufacture's guidelines. The Annexin V fluorescence was quantified with Flow Cytometry (BD FACSDiva Software v6). The percentage of Annexin V-positive cells was analyzed by FlowJo (Version 7.6.4). All the experiments were performed in triplicates.

\section{Ovarian cancer xenograft}

Six-week-old severe combined immunodeficiency (SCID) mice were obtained from the UCLA Division of Laboratory Animal Medicine. The mice were inoculated with $100 \mu \mathrm{l}(50 \%$ Matrigel/PBS) SKOV3 cell suspension $\left(3 \times 10^{6}\right)$ to both dorsal flanks of the mice with 25 -gauge syringes. When the tumor size reached between 90 and $100 \mathrm{~mm}^{3}$, the mice were randomly divided into control, Rad001, ATO, and combination groups. Rad001 and ATO were delivered intraperitoneally at 1 and $2.5 \mathrm{mg} / \mathrm{kg}$ per day respectively. The tumors were measured every 4 days with a caliper. These tumor measurements were converted to tumor volume using the formula $\left(V=0.52 \times L \times W^{2}\right)$, where $W$ and $L$ are the smaller and larger diameters. Animal body weight was also weighed the same day when tumor size was measured. On day 32, all the mice were killed, tumors dissected, and collected. All the animal experiments were conducted according to the protocol approved by the University of California, Los Angeles Animal Research Committee (ARC).

\section{Statistical analysis}

The normalized isobologram analysis and bars were performed with CalcuSyn software (version 2.1, Biosoft) and Microsoft Excel 2003 respectively. With median effects model, described by Chou (2010), the multiple compound dose-effect calculations were performed. CI values of $<0.9,>0.9$ and $<1.2,>1.2$ was considered as being synergistic, additive, and antagonistic respectively. Statistical analysis was performed by two-sided $t$-test. $P$ value $<0.05$ was regarded to be statistically significant.

The statistical significance of the differences in tumor volumes between treatment and control groups were determined by one-way ANOVA followed by the Dunnett's test. Statistical analyses of body weight were performed by one-way ANOVA followed by Tukey's test. The level of significance was set at $P<0.05$. Statistical calculations were performed using the SPSS version 13.0.

\section{Results}

\section{Synergy of Rad001 and ATO in reducing viability of SKOV3 and OV2008 ovarian cancer cell lines}

$\mathrm{PI} 3 \mathrm{~K} / \mathrm{AKT} / \mathrm{mTOR}$ signaling module plays a central role in many human cancers including ovarian cancer. However, inhibitors of this pathway such as Rad001 have limited therapeutic efficacy for ovarian cancer as a single agent (Liu et al. 2009), likely due to 
activation of compensatory pathways, which has been widely reported (Bartholomeusz \& Gonzalez-Angulo 2012). Recent preclinical studies have demonstrated that ATO can inhibit the growth of a variety of tumors in various models (Goussetis et al. 2010, Smith et al. 2010, Chiu et al. 2011, MartinezOutschoorn et al. 2011). As a high concentration of ATO is required, it can cause significant side effects, limiting its clinical utility.

If two drugs can synergize with each other, it is possible to achieve superior efficacy while decreasing side effects. Therefore, we tested whether Rad001 and ATO could synergize to inhibit the growth of ovarian cancer cells. As shown in Fig. 1A, the combination of these two agents can decrease cell number by $>50 \%$ than each agent alone in both SKOV3 and OV2008 cells, two of the most commonly used ovarian cancer cell lines. To confirm that the observed effect on cell number by the combination of Rad001 and ATO is truly through synergy, we performed a detailed, systematic study using each of the two compounds at varying concentrations and monitored ATP content as a surrogate of cell number. As shown in Fig. 1B, dosedependent decrease of cell number as measured by ATP contents was seen in SKOV3 and OV2008 cells when the cells were treated with either Rad001 or ATO. When these cells were treated with the combination of the two compounds, there was significantly more decrease in relative cell luminescence units in both cell lines (Z-axis) compared with treatment with either Rad001 or ATO alone (Fig. 1B). CI, a measure of synergistic activity of two compounds, was determined with the CalcuSyn software program, which confirmed significant synergism of the two compounds (Fig. 1C). For example, for SKOV3 cells, treatment with either $0.37 \mu \mathrm{M}$ Rad001 or $11.19 \mu \mathrm{M}$ ATO alone resulted in 12.71 and $31.51 \%$ reduction in cell number respectively. However, there was $67.60 \%$ reduction of cell number when the two compounds were combined $(\mathrm{CI}=0.76)$. MixtureAlgebraic estimate analysis, performed with CalcuSyn software, showed a strong synergy for most of the combinations of two compounds at various concentrations for the two cell lines (data not shown), suggesting that synergy is not limited to a particular threshold concentration of either of the two compounds.

\section{Combination of Rad001 and ATO synergistically induces cell death through apoptosis in SKOV3 and OV2008 ovarian cancer cells}

We performed trypan blue staining of the cells after treatment with each agent alone or in combination. As shown in Fig. 2A, there was a significant decrease in live cells (trypan blue negative) in each cell line after combination treatment compared with singlecompound treatment $(P<0.05)$, suggesting that the combination treatment induced a significant reduction of cell viability. This was further confirmed with the analysis of proteins involved in apoptotic cell death. When SKOV3 and OV2008 cells were treated with vehicle (DMSO), Rad001, ATO, or their combination for $18 \mathrm{~h}$, there was a significant increase in the cleaved form of apoptosis marker PARP in cells that received combination treatment (Fig. 2B). This was accompanied by the induction of cleaved form of caspase- 3 as well as the induced activity of caspase-3/7 (Fig. 2C), suggesting that apoptotic pathway was activated in response to the combination treatment. As shown in Fig. 2D, combination of Rad001 and ATO increased the fraction of apoptotic cells more significantly than single-agent treatment. Thus, we conclude that combination of Rad001 and ATO can result in synergistic cytotoxicity through activation of the apoptotic pathway.

\section{Combination of Rad001 and ATO synergistically induces autophagy in SKOV3 and OV2008 ovarian cancer cells}

It has been reported that Rad001 and ATO can each induce autophagy (Mabuchi et al. 2007a,b, Smith et al. 2010). Therefore, we next determined whether combination of the two drugs synergistically inhibits ovarian cancer cells through increased autophagy, as autophagy can also result in cell death (type II cell apoptosis). A hallmark of autophagy is the enhanced conversion of microtubule-associated protein 1 light chain 3 (LC3-1) to its faster-migrating form LC3-2. Another measure of autophagy is the size and number of p62 bodies and the levels of p62 protein, a polyubiquitin-binding protein that is thought to facilitate autophagic degradation of ubiquitinated protein aggregates. Inhibition of autophagy leads to an increase while induction of autophagy results in a decrease of p62 protein (Bjorkoy et al. 2005). As shown in Fig. 3A, there was substantially more LC3-2 after combination treatment compared with treatment with either Rad001 or ATO alone, and this was accompanied by a decrease in p62 protein level, suggesting that the combination of Rad001 and ATO can synergistically induce autophagy. We also examined the abundance of autophagosome in the tumor cells. Autophagosomes are characterized by membranous structures with double or multiple membrane layers. Transmission electron microscopic analysis of the cells demonstrated that the cells treated 
A

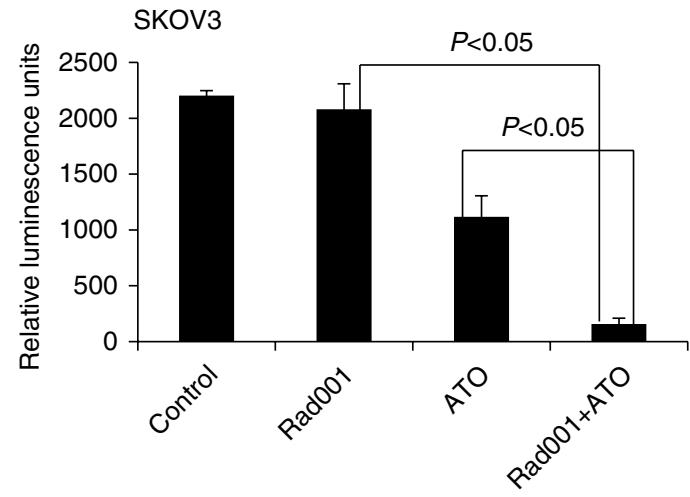

B $\quad \mathrm{SKOV} 3$

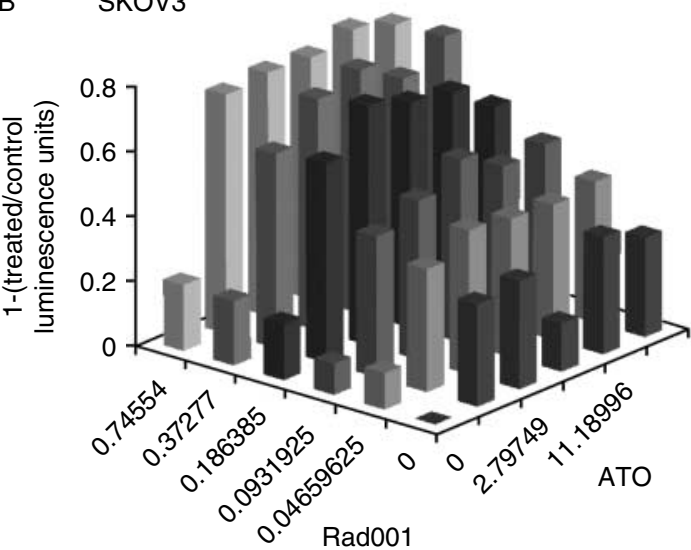

C

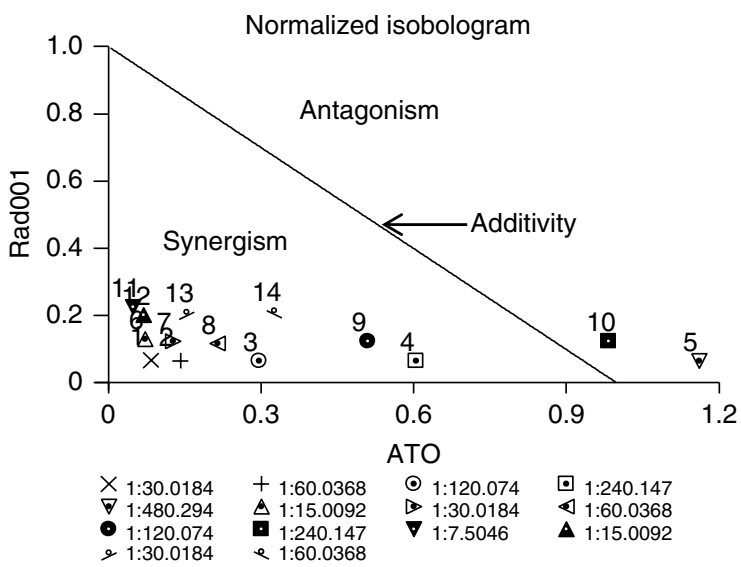

OV2008
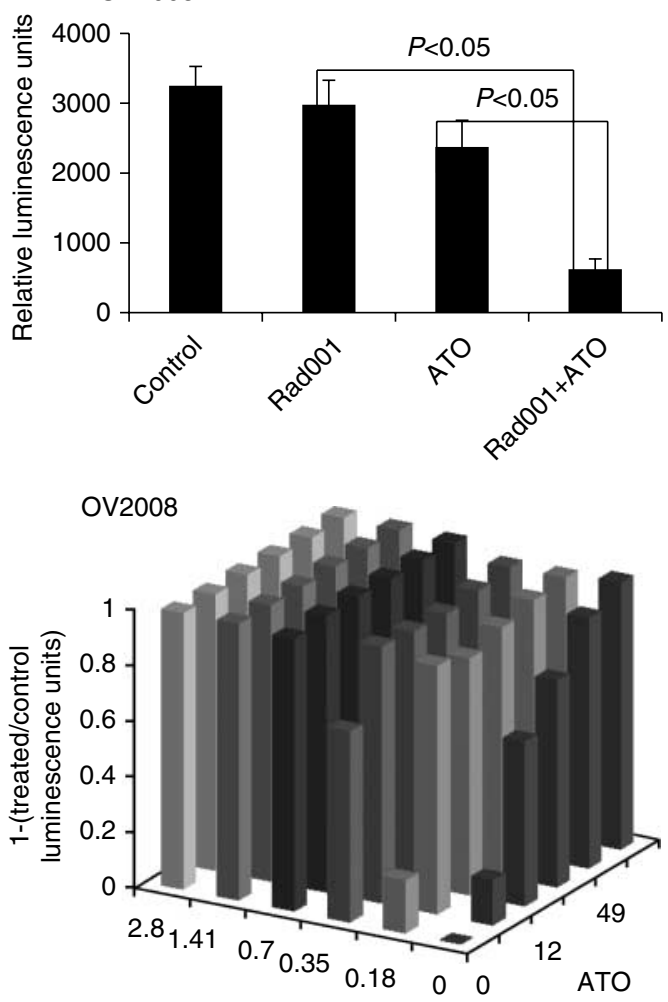

Rad001

OV2008

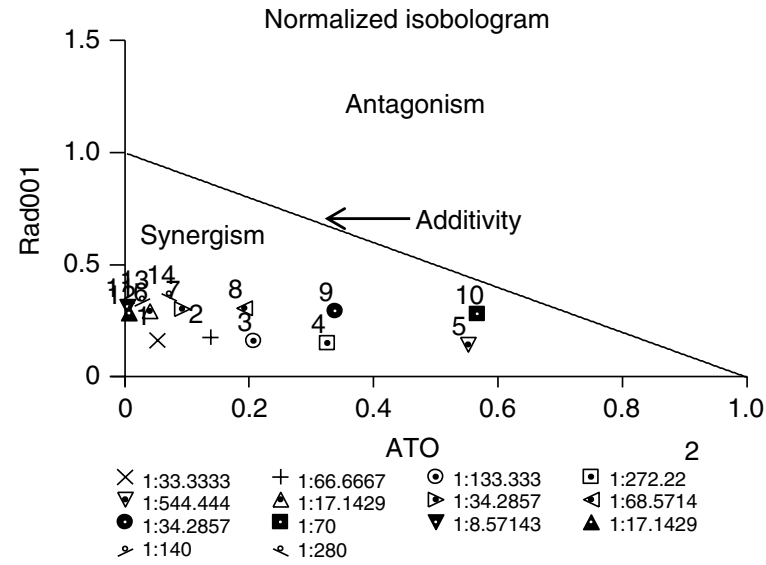

Figure 1 The combination of Rad001 and ATO induces a synergistic reduction of cell number (measured in luminescence units) in ovarian cancer cells. (A) ATO can collaborate with Rad001 to reduce cell number in SKOV3 and OV2008 cells. Rad001 (0.3 $\mu$ M), ATO $(12 \mu \mathrm{M})$, or their combination was used to treat SKOV3 or OV2008 cells for $18 \mathrm{~h}$ followed by Celltiter Glo assay to measure cell number. (B) A gradient dose of Rad001, ATO alone, and in combination was used to measure cell luminescence units as in A ( $X$-axis: Rad001 $\mu \mathrm{M}$, Y-axis: ATO $\mu \mathrm{M}, Z$-axis: 1-(treated luminescence unit/control luminescence unit)). Each bar in this 3D display represents relative cell loss in response to the compound treatment alone or in combination. A complete cell loss is equal to 1 , while the value for cells without drug treatment is set to 0 . A higher bar indicates a more severe cell loss. In this manner, it is visually clear that there is more severe cells loss with higher concentrations of the compounds and their combination. (C) Normalized isobologram analysis shows synergistic interactions of the two compounds in ovarian cancer SKOV3 and OV2008 cell lines. Normalized isobologram assays were performed with CalcuSyn software, which calculates the dose effect with the median effect method described by Chou (2010). Depending upon the location of the bar on the isobologram, combinations of the compounds can result in cell death in a synergistic, additive, or antagonistic manner as outline in the graph. A large number of combination groups of Rad001 and ATO are below the line, indicating synergistic killing of the ovarian cancer cells by the two compounds. 

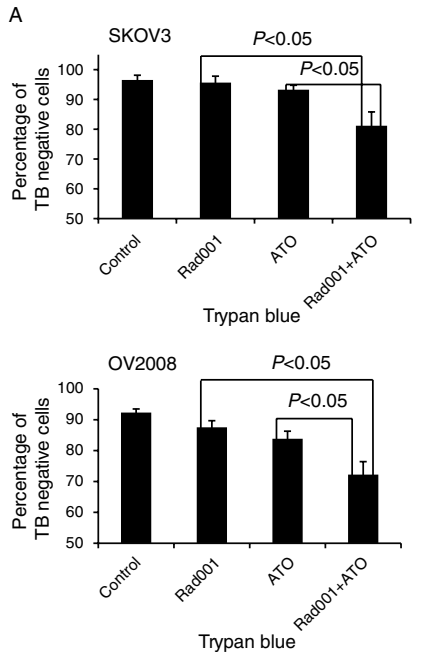

B

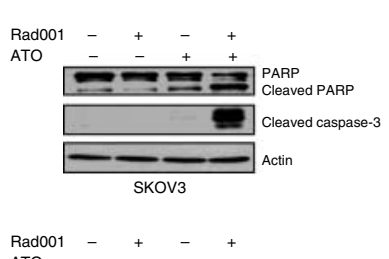

Rad00

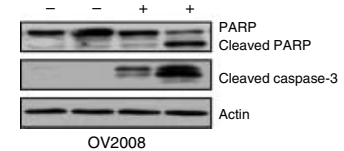

C
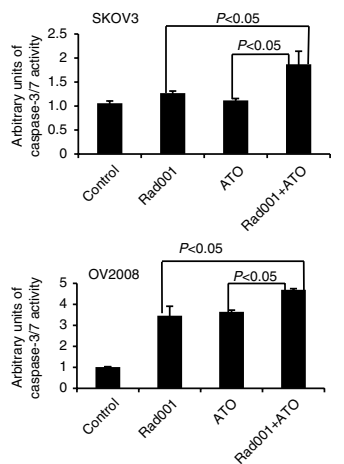
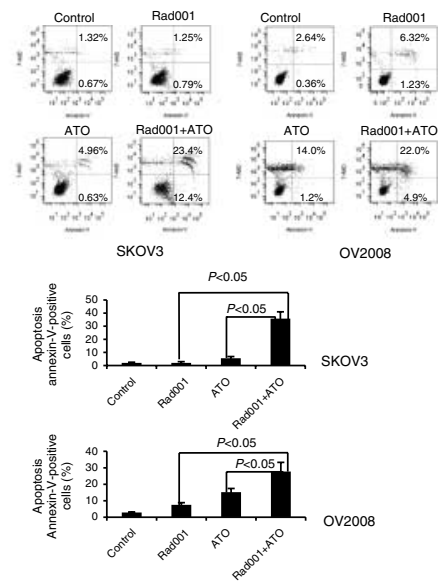

Figure 2 Combination of Rad001 and ATO synergistically reduces cell viability in ovarian cancer cells. SKOV3 cells were treated with DMSO (control), Rad001 $(0.37 \mu \mathrm{M})$, ATO $(11.19 \mu \mathrm{M})$, or their combination for $18 \mathrm{~h}$. OV2008 cells were treated with DMSO (control), Rad001 $(0.35 \mu \mathrm{M})$, ATO $(12.00 \mu \mathrm{M})$, or their combination for $18 \mathrm{~h}$. (A) Trypan blue analysis of SKOV3 and OV2008 cells that were treated alone or in combination. Combination treatment resulted in more reduction of the cell viability than either drug alone $(P<0.05)$. (B) Increased levels of cleaved PARP and cleaved caspase-3 were detected in SKOV3 and OV2008 cells after treatment with the combination of Rad001 and ATO for $18 \mathrm{~h}$. Lysates from cells treated with Rad001, ATO, and their combinations were separated by SDS-PAGE followed by immunoblot with respective antibodies. (C) Higher activities of caspase-3/7 were observed in SKOV3 and OV2008 cells treated with Rad001/ATO combination for $18 \mathrm{~h}$ than either drug alone $(P<0.05$ between the two groups). (D) Analysis of apoptosis was done with Annexin-V and 7-AAD staining flow cytometry assay. Right panels (top and bottom) show percentage of Annexin-V-positive cells. A significant increase in the percentage of apoptotic cells were observed with the combination treatment compared with single agent treatment in both SKOV3 and OV2008 cells $(P<0.05)$.

with the two compounds in combination had significantly increased number of vacuoles in comparison to single-agent treatment (Fig. 3B), again confirming that there was a synergistic induction of autophagy. The quantification of this increase is shown in Fig. 3C.

Autophagosomes can also be visualized through the expression of GFP-LC3, which upon autophagosome formation becomes clustered on the membrane vesicles and visible as a ring structure under the fluorescent microscope. This analysis as shown in Fig. 3D demonstrates that there were more punctuate GFPpositive vesicles in response to the combination treatment compared with single-agent treatment in both SKOV3 and OV2008 cells (lower panel of Fig. 3D). Consistent with the increase of autophagy in response to the combination treatment, we also found that Atg5-Atg12 conjugate, which is involved in the first of two ubiquitination-like reactions that control autophagy, was increased in the two cell lines treated with the two compounds simultaneously (Fig. 4A). Therefore, we conclude that there was a synergistic induction of autophagy in response to the combinatorial treatment of Rad001 and ATO, likely leading to autophagic cell death.

\section{Combination of Rad001 and ATO decreases the expression of $p-A K T$ and $p-4 E B P 1$, which are critical for autophagy and apoptosis}

Autophagy is a type of programed cell death important in preventing cell aging and providing the energy required for cell survival and repair during environmental stresses such as hypoxia, infection, and nutrient starvation (Jin \& White 2007, Chen \& KarantzaWadsworth 2009, Lozy \& Karantza 2012). Two main pathways that regulate autophagy in response to starvation are the class I PI3K/AKT/mTOR signaling pathway and the mitogen-activated protein kinase (MAPK)/extracellular signal-regulated kinase (ERK) signaling pathway. The activation of AKT/mTOR pathway has been negatively associated with autophagy (Jin \& White 2007, Chen et al. 2010). To determine whether reduction of phospho-AKT might be responsible for the induction of autophagy in the above experiments, we examined the expression level of AKT as well as its activation status as reflected by the phosphorylation of AKT. As shown in Fig. 4B, there was substantially less $\mathrm{p}$-AKT in response to the combination treatment in comparison with singleagent treatment. It is known that inhibition of mTOR 
A

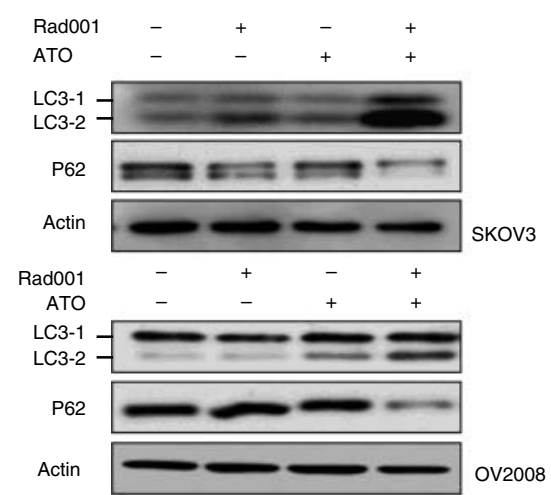

C

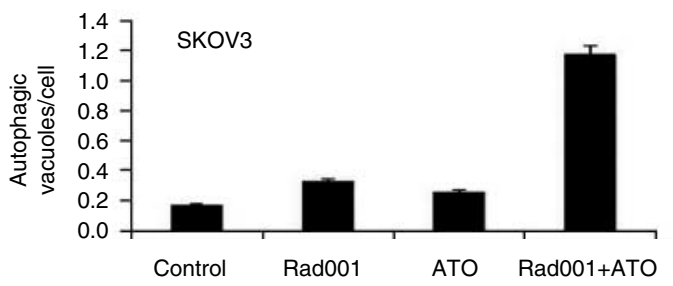

D
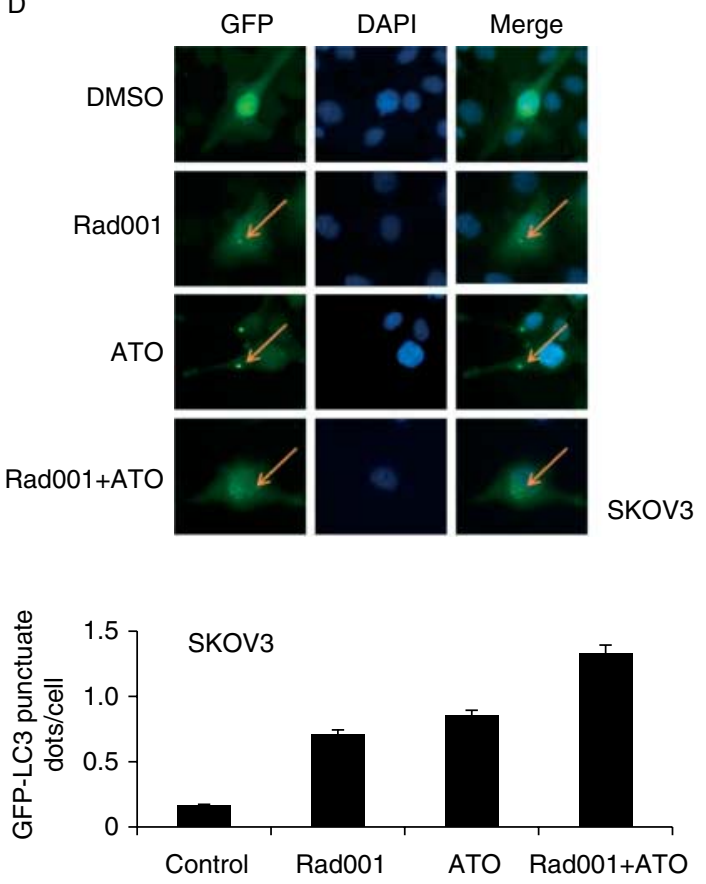
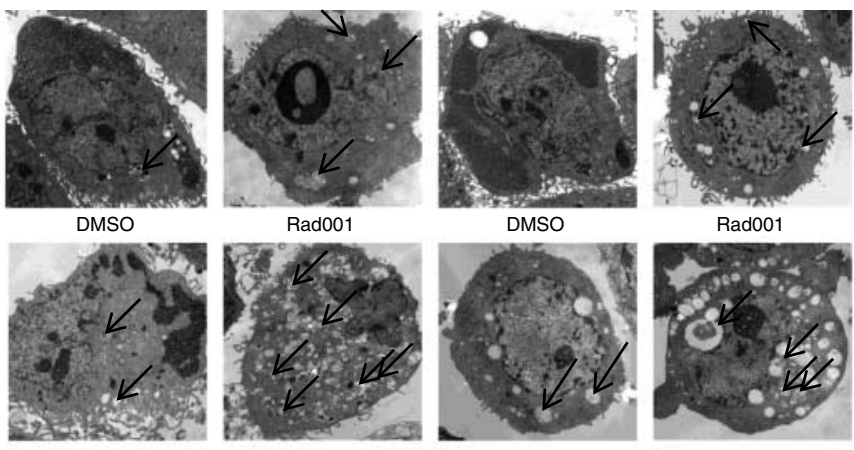

ATO

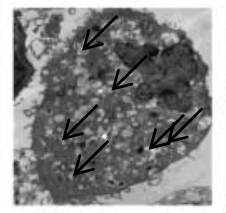

Rad001+ATO SKOV3
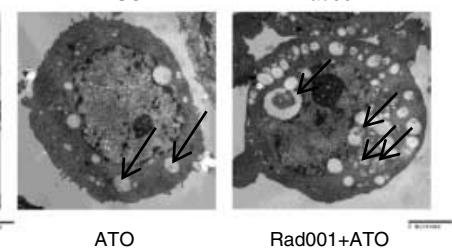

OV2008
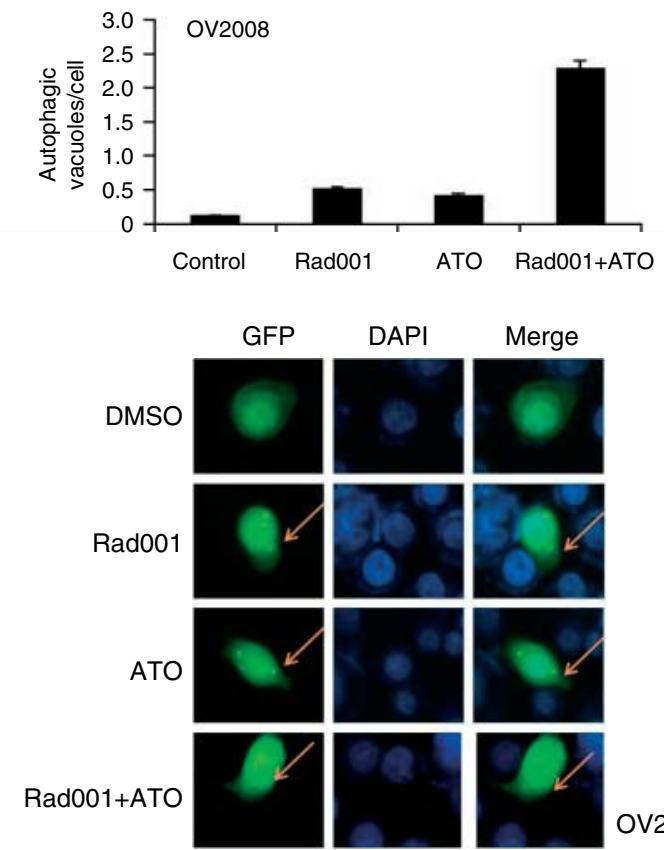

OV2008

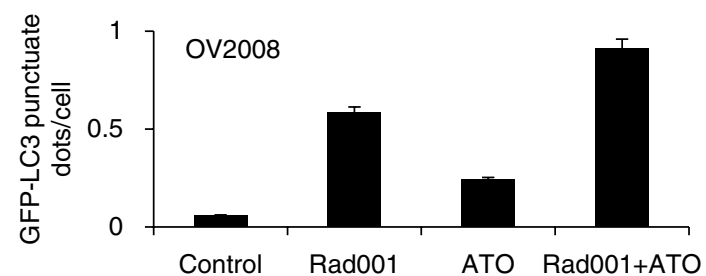

Figure 3 Rad001 and ATO combination synergistically induces autophagy in SKOV3 and OV2008 cells. Autophagy was analyzed after $18 \mathrm{~h}$ of drug treatment. SKOV3 cells were treated with DMSO (control), Rad001 $(0.37 \mu \mathrm{M})$, ATO $(11.19 \mu \mathrm{M})$, or their combination. OV2008 cells were treated with DMSO (control), Rad001 $(0.35 \mu \mathrm{M})$, ATO $(12.00 \mu \mathrm{M})$, or their combination.

(A) Upregulation of LC3-2 and downregulation of P62 in SKOV3 and OV2008 cells treated with combination of two compounds. (B) More autophagosomes (arrows) were observed in the electron microscopic images from cells treated with Rad001/ATO combination than each drug alone. (C) The number of autophagic vacuoles/cell was quantified in cells treated with control, Rad001, ATO, and their combination. Thirty cells were counted in each group. (D) Autophagosome analysis through GFP-LC3 expression. The cells were transfected with GFP-LC3 plasmid and treated with single drug or their combination. The cells were then fixed with paraformaldehyde and visualized with epifluorescence. Yellow arrows indicate the punctuate pattern of GFP-LC3, representative of autophagosome, and nuclei were visualized through DAPI staining. The number of GFP-LC3 punctuate dots/cell was quantified with 50 GFP + cells in each group in the lower panel. 
by Rad001 can lead to compensatory activation of AKT through upregulation of IGF1R/PI3K pathway (Serra et al. 2011). It appears that addition of ATO can repress the compensatory activation of AKT, resulting in sustained inhibition of the pathway. Consistent with this observation, we found that there was profound synergistic reduction of phospho-4E-BP1, which is a downstream target of mTOR in both cell lines. We did not observe similar inhibition of other targets of mTOR such as p70S6K and eIF4E, which may have different mechanisms of regulation.

ERK1/ERK2 (also known as p42/p44MAPK respectively and officially named MAPK 1 and 3) are two isoforms of ERK that belong to the family of MAPKs, which include ERK5, the JNK1/2/3, and the p38 MAP kinases (p38 a,b,d,c). These enzymes are activated through a sequential phosphorylation cascade that amplifies and transduces signals from the cell membrane to the nucleus (Martin \& Pognonec 2009). It has been shown that constitutive activation of ERK by active Raf, cadmium, or IGF1 receptor induces autophagic programed cell death (Cagnol \& Chambard 2009). In addition, ERK activity has been associated with autophagy and autophagic cell death in many cancer cell lines in response to different stresses, such as aurintricarboxylic acid in human colorectal cancer cell line HT29, soyasaponins in human colon adenocarcinoma HCT-15, and L929 cells (Pattingre et al. 2003, Bjorkoy et al. 2005). Conversely, in human ovarian cancer cells, cytoplasmic sequestration of ERK by PEA-15 has been shown to promote autophagy (Bartholomeusz et al. 2008). We studied ERK in our experimental system and as shown in Fig. 4D, there was a reduction of p-ERK1 expression in response to

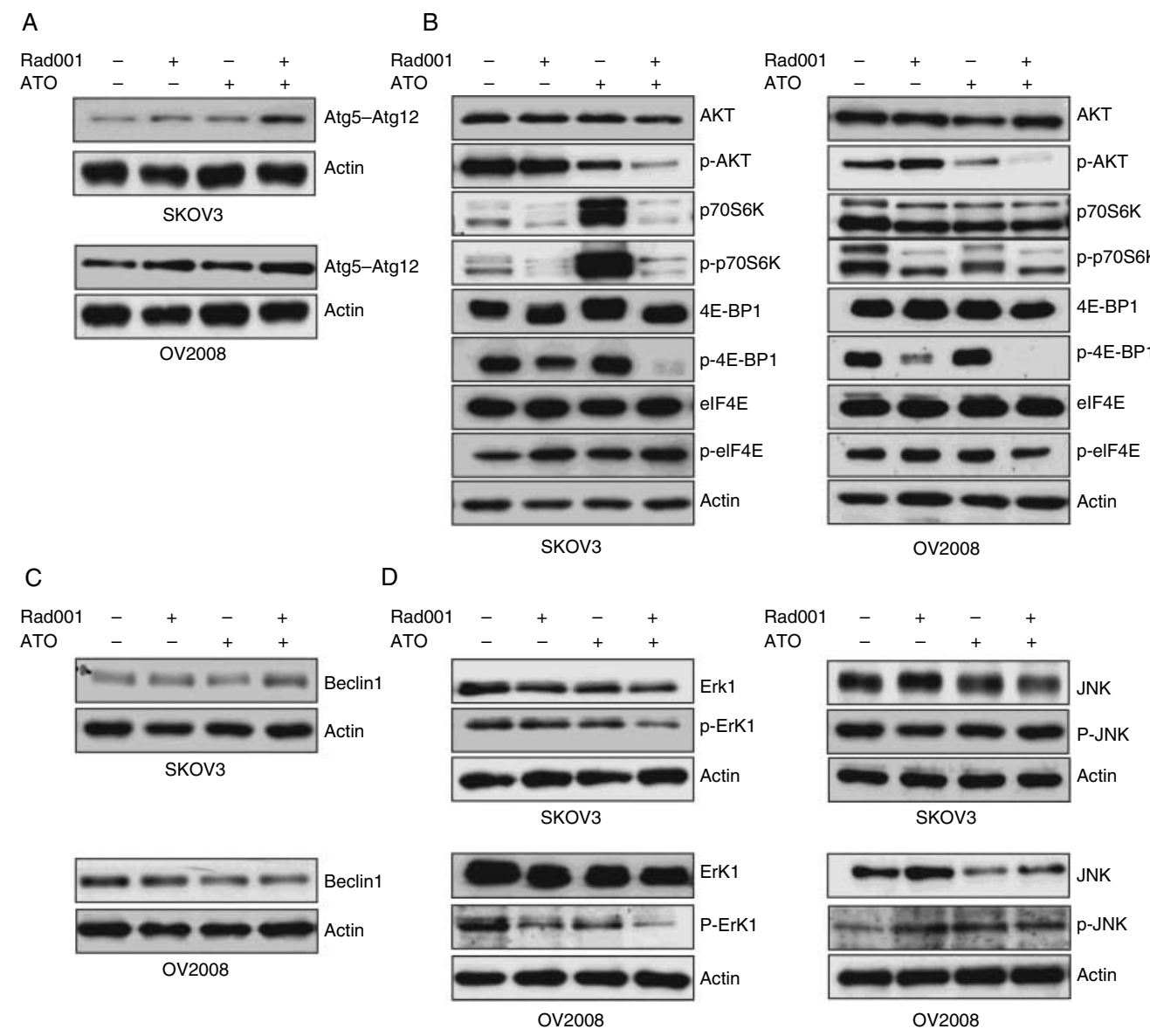

Figure 4 Combination of ATO and Rad001 induces apoptosis and autophagy in SKOV3 and OV2008 ovarian cancer cells. Combination treatment increased the levels of Atg5-Atg12 (A) and decreased the levels of p-AKT, p-4E-BP1 compared with Rad001 or ATO alone (B). Rad001 treatment decreased expression levels of $p$-p70S6K and p-4E-BP1. In SKOV3 cell line, ATO could induce the express of p-p70S6K protein level. p-elF4E protein level did not change significantly in response to the combination treatment in both cells. (C) Beclin1 (Atg6) level did not change in response to the combination treatment. (D) p-ERK1 protein level decreased more pronouncedly in response to the combination treatment than single-drug treatment. The p-JNK protein level was similar in different treatment groups. 
combination treatment compared with Rad001 or ATO alone in both the SKOV3 and the OV2008 cell lines. On the other hand, there was no significant change in JNK signaling pathway (Fig. 4D), suggesting that MAPK pathway is unlikely to be the cause of the induction of autophagy.

Beclin1 (Atg6) is critical for the initiation of autophagy pathway (Wirawan et al. 2012). However, as shown in Fig. 4C, there was no synergistic induction of Atg6 in the ovarian cancer cell lines in response to the combination treatment, suggesting that the induction of autophagy is not merely a response to increased level of the initiator of autophagy.

In summary, Rad001 and ATO combination treatment of ovarian cancer cells results in synergistic induction of autophagy and apoptosis likely as a result of sustained inhibition of AKT-mTOR pathway as well as inhibition of specific downstream targets such as $4 \mathrm{E}-\mathrm{BP} 1$.

\section{Rad001 and ATO combination inhibited growth of ovarian cancer xenograft}

We next determined whether the synergy of ATO and Rad001 exists in a preclinical ovarian cancer xenograft model. We initiated tumor growth with s.c. injection of SKOV3 cells on the flanks of SCID mice. When tumor size reached $\sim 100 \mathrm{~mm}^{3}$, we started daily i.p. injection of the two compounds followed by measurement of tumor sizes and body weight on every fourth day (6 tumors/group). We used ATO at a dose of $2.5 \mathrm{mg} / \mathrm{kg}$ per day and Rad001 at a dose of $1 \mathrm{mg} / \mathrm{kg}$ per day according to the literature (Pattingre et al. 2003, Mabuchi et al. 2007b, Aide et al. 2010). As shown in Fig. 5A, combination of Rad001 and ATO more significantly inhibited SKOV3 xenograft tumor growth than treatment with either compound alone $(* P<0.05$ compared with untreated animals and $* * P<0.05$ compared with Rad001-treated animals or ATOtreated animals). On the other hand, as shown in Fig. 5B, body weights were not significantly different among the different groups $(* P>0.05$ compared with untreated animals and $* * P>0.05$ compared with Rad001-treated animals or ATO-treated animals), suggesting little side effects. No complications such as anaphylaxis and skin necrosis were observed throughout the course of the study. We also examined the autophagosome formation in the xenograft tumors by transmission electron microscopic analysis. Consistent with the in vitro results, combination of the two compounds increased autophagosome formation in the xenograft tumors (Fig. 5C). The increase of autophagy was also confirmed at the molecular level by the increase of LC3-1 and LC3-2 levels in the combination treated tumors compared with tumors that received single compound treatment (Fig. 5D). These results strongly suggest that Rad001 and ATO synergize with each other to inhibit ovarian cancer growth, establishing a foundation for future clinical testing in patients.

\section{Discussion}

Many novel agents have been developed that targetspecific molecular pathways that are responsible for the malignant behavior of tumor cells for increased therapeutic efficacy and minimal side effects. However, some do not show clinical efficacy and for others resistance commonly occurs after initial therapeutic benefits. A common cause for the above observations is that tumor cells activate compensatory pathways rendering the inhibitor ineffective (Dong et al. 2010). Combination therapies targeting multiple pathways simultaneously have the potential to overcome this problem and benefit cancer patients.

Ovarian cancer represents some of the deadliest human malignancies. Although PI3K/AKT/mTOR pathway has been shown to be an important growth stimulatory pathway, mTOR inhibitors such as Rad001 have not been shown to be useful clinically as single agents (O'Reilly \& McSheehy 2010). Therefore, we searched for agents that may synergize with Rad001 in killing ovarian cancer cells, focusing on those that are already in clinical use. We found that ATO, a drug that has been clinically useful for certain type of leukemia, can synergize with Rad001 and increase apoptosis and autophagy appear to be responsible for their synergistic effect.

In recent decades, studies in the field of cell death have focused on understanding the molecular mechanisms of apoptosis. In addition to canonical apoptosis (type I apoptosis) and necrosis, there is evidence that cells can die through alternative pathways. Autophagic or type II cell death, characterized by abundant autophagic vacuoles in the cytoplasm of dying cells, has been described in recent years (Lin et al. 2010). Type II cell death occurs because of persistent autophagy with excessive degradation of cellular components essential for survival (Ciuffreda et al. 2010). Two major pathways that regulate autophagy in mammalian cells are the PI3K-AKT-mTOR pathway and the AMP-activated protein kinase (AMPK) pathway. The PI3K-AKTmTOR pathway is activated by the binding of growth factors such as insulin-like growth factors 1 (IGF1) to their receptor. Activated PI3K converts PIP2 to PIP3 to 

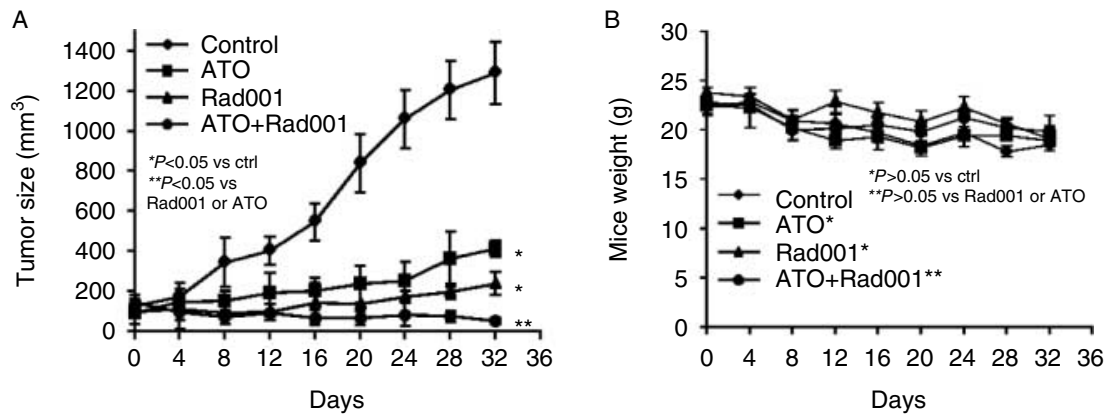

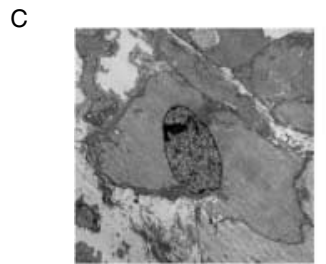

Control

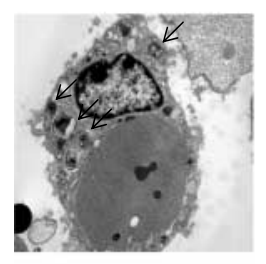

ATO

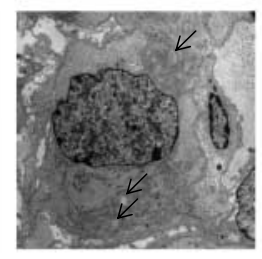

Rad001

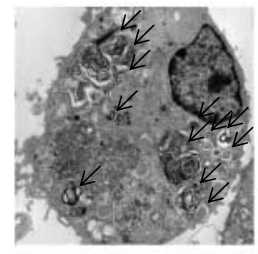

Rad001+ATO

Figure 5 Combination of Rad001 and ATO significantly inhibits the growth of SKOV3 cells xenograft in a mouse model. (A) Antitumor activity on SKOV3 xenografts by various compounds. Mice were treated (i.p.) with Rad001 at $1 \mathrm{mg} / \mathrm{kg}$, ATO at $2.5 \mathrm{mg} / \mathrm{kg}$, or their combination daily. The tumor sizes were measured every 4 days $\left({ }^{\star} P<0.05\right.$ compared with untreated animals and ${ }^{\star \star} P<0.05$ compared with Rad001-treated animals or ATO-treated animals). (B) The body weights of the mice were measured every 4 days ( ${ }^{\star} P>0.05$ compared with untreated animals and ${ }^{* \star} P>0.05$ compared with Rad001-treated animals or ATO-treated animals).

(C) Electron microscopic analysis of the tumors collected from various treatment groups. Arrows indicate autophagosomes. Quantitation of the number of autophagosomes in 30 cells from the xenograft is shown on the right panel. (D) Immunoblot analysis for LC3 from the lysate prepared from the xenograft tumors from mice that were treated with single agents or their combination.

activate AKT. AMPK is normally activated by its upstream kinase LKB-1 or by an increased intracellular ratio of AMP/ATP. Active AMPK directly phosphorylates TSC2 and inhibits mTOR to activate autophagy. Activated mTOR phosphorylates 4EBP-1 and p70S6K, which lead to protein translation and tumor growth (Kim et al. 2008, Noske et al. 2008, Schmid et al. 2010).

Cytoreductive surgery and empirical combination of chemotherapeutics have improved 5-year survival for ovarian cancer patients but have not increased the overall rate of cure. Poor outcomes are at least in part due to delay in diagnosis and to the subpopulations of ovarian cancer cells that become resistant to conventional chemotherapeutic drugs (Karam \& Dorigo 2012). With the increased understanding of the molecular, cellular, and clinical biology of ovarian cancer comes the hope of personalized therapy with molecularly targeted agents (Friday \& Adjei 2008, Bast 2011). Activation of the PI3K pathway is observed in more than one-third of high-grade serous ovarian cancers. A small fraction of cancers contain activating mutations of $P I K 3 C A$, inactivating mutations of PTEN, or amplification of AKT, and a larger fraction exhibits autocrine/paracrine growth factor stimulation (Bast 2011). PI3K activity prevents apoptosis and increases drug resistance (Lin et al. 2001). PI3K inhibitors slow the growth of human ovarian cancer xenografts and enhance paclitaxel response (Uddin et al. 2010). Currently, inhibitors of mTOR, AKT, and PI3K are being evaluated in a variety of tumor types. However, because of compensatory activation of other signaling molecules, inhibition of multiple pathways will be required to achieve better efficacy.

ATO is a metalloid that exhibits potent antineoplastic effects in vitro and in vivo (Platanias 2009). It is a potent inducer of autophagy in leukemia cells. The induction of autophagy appears to require 
activation of the MEK/ERK pathway but not the AKT/ mTOR or JNK pathways (Goussetis et al. 2010). ATO induces autophagy and apoptosis in human glioma cells in vitro and in vivo through downregulation of survivin (Chiu et al. 2011), but significant side effects limit its clinical utility. Our finding that ATO can synergize with Rad001 through induction of autophagic cell death provides us with a novel therapeutic modality to increase the efficacy and reduce the side effects of existing drugs. An example similar to this is the combination treatment of fibrosarcoma with ATO and irradiation (Chiu et al. 2010).

The main finding of our study is that combination of ATO and Rad001 induces apoptosis of ovarian cancer cells in a synergistic manner and enhanced autophagy is likely a major underlying mechanism. Apoptosis has long been established as a form of programed cell death and is known to be a key component of tumor suppression. The apoptotic pathways are well studied, and the role of autophagy in modulating apoptosis has been convincingly documented in vivo. For example, overexpression of wild-type Atg1 in Drosophila elicits a strong autophagic response, and cells that have high levels of Atg1 are selectively and rapidly eliminated (Berry \& Baehrecke 2007, 2008, Dutta \& Baehrecke 2008, Batlevi et al. 2010). The co-activation of autophagy and apoptosis has also been observed in cancer cells (Gao et al. 2010, Lian et al. 2010, Liang 2010, Rubinstein et al. 2011, Zhou et al. 2011). Simultaneous induction of autophagy and apoptosis likely leads to cell death in response to the combination treatment of histone deacetylase inhibitor and the Bcl-2 homology domain-3 mimetic GX15-070 in leukemic cells (Wei et al. 2010). Our results demonstrate that ATO can synergize with Rad001 to enhance autophagy and induce cell death in vitro and in a xenograft model. Such novel combinatorial chemotherapeutics may represent a new direction to develop therapies for ovarian cancer.

\section{Declaration of interest}

The authors declare that there is no conflict of interest that could be perceived as prejudicing the impartiality of the research reported.

\section{Funding}

J Huang is supported by UCLA SPORE in Prostate Cancer (PI: Robert Reiter), Department of Defense Prostate Cancer Research Program grants W81XWH-11-1-0227 (PI: J Huang) and W81XWH-12-1-0206 (PI: Lily Wu), and National Cancer Institute (1R01CA158627-01; PI: Leonard Marks).

\section{Acknowledgements}

The authors thank Dr Oliver Dorigo for the cell lines used in this study.

\section{References}

Aide N, Kinross K, Cullinane C, Roselt P, Waldeck K, Neels O, Dorow D, McArthur G \& Hicks RJ 2010 18F-FLT PET as a surrogate marker of drug efficacy during mTOR inhibition by everolimus in a preclinical cisplatinresistant ovarian tumor model. Journal of Nuclear Medicine 51 1559-1564. (doi:10.2967/jnumed.109. 073288)

Bartholomeusz C \& Gonzalez-Angulo AM 2012 Targeting the PI3K signaling pathway in cancer therapy. Expert Opinion on Therapeutic Targets 16 121-130. (doi:10.1517/14728222.2011.644788)

Bartholomeusz C, Rosen D, Wei C, Kazansky A, Yamasaki F, Takahashi T, Itamochi H, Kondo S, Liu J \& Ueno NT 2008 PEA-15 induces autophagy in human ovarian cancer cells and is associated with prolonged overall survival. Cancer Research 68 9302-9310. (doi:10.1158/0008-5472.CAN08-2592)

Bast RC Jr 2011 Molecular approaches to personalizing management of ovarian cancer. Annals of Oncology 22 (Suppl 8) viii5-viii15. (doi:10.1093/annonc/mdr516)

Batlevi Y, Martin DN, Pandey UB, Simon CR, Powers CM, Taylor JP \& Baehrecke EH 2010 Dynein light chain 1 is required for autophagy, protein clearance, and cell death in Drosophila. PNAS 107 742-747. (doi:10.1073/pnas. 0907967107)

Berry DL \& Baehrecke EH 2007 Growth arrest and autophagy are required for salivary gland cell degradation in Drosophila. Cell 131 1137-1148. (doi:10.1016/j.cell. 2007.10.048)

Berry DL \& Baehrecke EH 2008 Autophagy functions in programmed cell death. Autophagy 4 359-360.

Bjorkoy G, Lamark T, Brech A, Outzen H, Perander M, Overvatn A, Stenmark H \& Johansen T 2005 p62/SQSTM1 forms protein aggregates degraded by autophagy and has a protective effect on huntingtininduced cell death. Journal of Cell Biology 171 603-614. (doi:10.1083/jcb.200507002)

Cagnol S \& Chambard JC 2009 ERK and cell death: mechanisms of ERK-induced cell death - apoptosis, autophagy and senescence. FEBS Journal 277 2-21. (doi:10.1111/j.1742-4658.2009.07366.x)

Campone M, Levy V, Bourbouloux E, Berton Rigaud D, Bootle D, Dutreix C, Zoellner U, Shand N, Calvo F \& Raymond E 2009 Safety and pharmacokinetics of paclitaxel and the oral mTOR inhibitor everolimus in advanced solid tumours. British Journal of Cancer 100 315-321. (doi:10.1038/sj.bjc.6604851)

Chen N \& Karantza-Wadsworth V 2009 Role and regulation of autophagy in cancer. Biochimica et Biophysica Acta 1793 1516-1523. (doi:10.1016/j.bbamcr.2008.12.013) 
Chen S, Rehman SK, Zhang W, Wen A, Yao L \& Zhang J 2010 Autophagy is a therapeutic target in anticancer drug resistance. Biochimica et Biophysica Acta 1806 220-229.

Chiu HW, Lin JH, Chen YA, Ho SY \& Wang YJ 2010 Combination treatment with arsenic trioxide and irradiation enhances cell-killing effects in human fibrosarcoma cells in vitro and in vivo through induction of both autophagy and apoptosis. Autophagy 6 353-365. (doi:10.4161/auto.6.3.11229)

Chiu HW, Ho YS \& Wang YJ 2011 Arsenic trioxide induces autophagy and apoptosis in human glioma cells in vitro and in vivo through downregulation of survivin. Journal of Molecular Medicine 89 927-941. (doi:10.1007/s00109-011-0763-1)

Chou TC 2010 Drug combination studies and their synergy quantification using the Chou-Talalay method. Cancer Research 70 440-446. (doi:10.1158/0008-5472.CAN-091947)

Ciuffreda L, Di Sanza C, Incani UC \& Milella M 2010 The mTOR pathway: a new target in cancer therapy. Current Cancer Drug Targets 10 484-495. (doi:10.2174/ 156800910791517172)

Dong Y, Li A, Wang J, Weber JD \& Michel LS 2010 Synthetic lethality through combined Notch-epidermal growth factor receptor pathway inhibition in basal-like breast cancer. Cancer Research 70 5465-5474. (doi:10.1158/0008-5472.CAN-10-0173)

Dutta S \& Baehrecke EH 2008 Warts is required for PI3Kregulated growth arrest, autophagy, and autophagic cell death in Drosophila. Current Biology 18 1466-1475. (doi:10.1016/j.cub.2008.08.052)

Friday BB \& Adjei AA 2008 Advances in targeting the Ras/Raf/MEK/Erk mitogen-activated protein kinase cascade with MEK inhibitors for cancer therapy. Clinical Cancer Research 14 342-346. (doi:10.1158/1078-0432. CCR-07-4790)

Gao P, Bauvy C, Souquere S, Tonelli G, Liu L, Zhu Y, Qiao Z, Bakula D, Proikas-Cezanne T, Pierron G et al. 2010 The Bcl-2 homology domain 3 mimetic gossypol induces both Beclin 1-dependent and Beclin 1-independent cytoprotective autophagy in cancer cells. Journal of Biological Chemistry 285 25570-25581. (doi:10.1074/jbc.M110.118125)

Goussetis DJ, Altman JK, Glaser H, McNeer JL, Tallman MS \& Platanias LC 2010 Autophagy is a critical mechanism for the induction of the antileukemic effects of arsenic trioxide. Journal of Biological Chemistry 285 29989-29997. (doi:10.1074/jbc.M109.090530)

Guertin DA \& Sabatini DM 2007 Defining the role of mTOR in cancer. Cancer Cell 12 9-22. (doi:10.1016/j.ccr.2007. 05.008)

Jin S \& White E 2007 Role of autophagy in cancer: management of metabolic stress. Autophagy 3 28-31.

Karam A \& Dorigo O 2012 MMPs in ovarian cancer as therapeutic targets. Anti-Cancer Agents in Medicinal Chemistry [in press].
Kim KW, Hwang M, Moretti L, Jaboin JJ, Cha YI \& Lu B 2008 Autophagy upregulation by inhibitors of caspase-3 and mTOR enhances radiotherapy in a mouse model of lung cancer. Autophagy 4 659-668.

Lian J, Karnak D \& Xu L 2010 The Bcl-2-Beclin 1 interaction in (-)-gossypol-induced autophagy versus apoptosis in prostate cancer cells. Autophagy 6 1201-1203. (doi:10.4161/auto.6.8.13549)

Liang C 2010 Negative regulation of autophagy. Cell Death and Differentiation 17 1807-1815. (doi:10.1038/cdd. 2010.115)

Lin MT, Lee RC, Yang PC, Ho FM \& Kuo ML 2001 Cyclooxygenase-2 inducing Mcl-1-dependent survival mechanism in human lung adenocarcinoma CL1.0 cells. Involvement of phosphatidylinositol 3-kinase/Akt pathway. Journal of Biological Chemistry 276 48997-49002. (doi:10.1074/jbc.M107829200)

Lin CI, Whang EE, Donner DB, Du J, Lorch J, He F, Jiang X, Price BD, Moore FD Jr \& Ruan DT 2010 Autophagy induction with RAD001 enhances chemosensitivity and radiosensitivity through Met inhibition in papillary thyroid cancer. Molecular Cancer Research 8 1217-1226. (doi:10.1158/1541-7786.MCR-10-0162)

Liu Q, Thoreen C, Wang J, Sabatini D \& Gray NS 2009 mTOR mediated anti-cancer drug discovery. Drug Discovery Today. Therapeutic Strategies 6 47-55. (doi:10.1016/j.ddstr.2009.12.001)

Lozy F \& Karantza V 2012 Autophagy and cancer cell metabolism. Seminars in Cell \& Developmental Biology 23 395-401. (doi:10.1016/j.semcdb.2012.01.005)

Mabuchi S, Altomare DA, Cheung M, Zhang L, Poulikakos PI, Hensley HH, Schilder RJ, Ozols RF \& Testa JR 2007a RAD001 inhibits human ovarian cancer cell proliferation, enhances cisplatin-induced apoptosis, and prolongs survival in an ovarian cancer model. Clinical Cancer Research 13 4261-4270. (doi:10.1158/1078-0432.CCR06-2770)

Mabuchi S, Altomare DA, Connolly DC, Klein-Szanto A, Litwin S, Hoelzle MK, Hensley HH, Hamilton TC \& Testa JR 2007b RAD001 (Everolimus) delays tumor onset and progression in a transgenic mouse model of ovarian cancer. Cancer Research 67 2408-2413. (doi:10.1158/ 0008-5472.CAN-06-4490)

Mabuchi S, Hisamatsu T, Kawase C, Hayashi M, Sawada K, Mimura K, Takahashi K, Takahashi T, Kurachi H \& Kimura T 2011 The activity of trabectedin as a single agent or in combination with everolimus for clear cell carcinoma of the ovary. Clinical Cancer Research 17 4462-4473. (doi:10.1158/1078-0432.CCR-10-2987)

Martin P \& Pognonec P 2009 ERK and cell death: cadmium toxicity, sustained ERK activation and cell death. FEBS Journal 277 39-46. (doi:10.1111/j.1742-4658.2009. 07369.x)

Martinez-Outschoorn UE, Goldberg A, Lin Z, Ko YH, Flomenberg N, Wang C, Pavlides S, Pestell RG, Howell A, Sotgia F et al. 2011 Anti-estrogen resistance in breast cancer is induced by the tumor microenvironment and can 
be overcome by inhibiting mitochondrial function in epithelial cancer cells. Cancer Biology \& Therapy 12 924-938. (doi:10.4161/cbt.12.10.17780)

Mazzoletti M, Bortolin F, Brunelli L, Pastorelli R, Di Giandomenico S, Erba E, Ubezio P \& Broggini M 2011 Combination of PI3K/mTOR inhibitors: antitumor activity and molecular correlates. Cancer Research 71 4573-4584. (doi:10.1158/0008-5472.CAN-10-4322)

Mondesire WH, Jian W, Zhang H, Ensor J, Hung MC, Mills GB \& Meric-Bernstam F 2004 Targeting mammalian target of rapamycin synergistically enhances chemotherapy-induced cytotoxicity in breast cancer cells. Clinical Cancer Research 10 7031-7042. (doi:10.1158/10780432.CCR-04-0361)

Nick AM, Stone RL, Armaiz-Pena G, Ozpolat B, Tekedereli I, Graybill WS, Landen CN, Villares G, Vivas-Mejia P, Bottsford-Miller J et al. 2011 Silencing of p130Cas in ovarian carcinoma: a novel mechanism for tumor cell death. Journal of the National Cancer Institute $\mathbf{1 0 3}$ 1596-1612. (doi:10.1093/jnci/djr372)

Noske A, Lindenberg JL, Darb-Esfahani S, Weichert W, Buckendahl AC, Roske A, Sehouli J, Dietel M \& Denkert C 2008 Activation of mTOR in a subgroup of ovarian carcinomas: correlation with p-eIF-4E and prognosis. Oncology Reports 20 1409-1417.

O'Reilly T \& McSheehy PM 2010 Biomarker development for the clinical activity of the mTOR inhibitor everolimus (RAD001): processes, limitations, and further proposals. Translational Oncology 3 65-79.

Pattingre S, Bauvy C \& Codogno P 2003 Amino acids interfere with the ERK1/2-dependent control of macroautophagy by controlling the activation of Raf-1 in human colon cancer HT-29 cells. Journal of Biological Chemistry 278 16667-16674. (doi:10.1074/jbc.M210998200)

Platanias LC 2009 Biological responses to arsenic compounds. Journal of Biological Chemistry 284 18583-18587. (doi:10.1074/jbc.R900003200)

Qian W, Liu J, Jin J, Ni W \& Xu W 2007 Arsenic trioxide induces not only apoptosis but also autophagic cell death in leukemia cell lines via up-regulation of Beclin-1. Leukemia Research 31 329-339. (doi:10.1016/j.leukres. 2006.06.021)

Rubinstein AD, Eisenstein M, Ber Y, Bialik S \& Kimchi A 2011 The autophagy protein Atg12 associates with antiapoptotic Bcl-2 family members to promote mitochondrial apoptosis. Molecular Cell 44 698-709. (doi:10.1016/j.molcel.2011.10.014)

Schmid K, Bago-Horvath Z, Berger W, Haitel A, Cejka D, Werzowa J, Filipits M, Herberger B, Hayden H \&
Sieghart W 2010 Dual inhibition of EGFR and mTOR pathways in small cell lung cancer. British Journal of Cancer 103 622-628. (doi:10.1038/sj.bjc.6605761)

Serra V, Scaltriti M, Prudkin L, Eichhorn PJ, Ibrahim YH, Chandarlapaty S, Markman B, Rodriguez O, Guzman M, Rodriguez $\mathrm{S}$ et al. $2011 \mathrm{PI} 3 \mathrm{~K}$ inhibition results in enhanced HER signaling and acquired ERK dependency in HER2-overexpressing breast cancer. Oncogene 30 2547-2557. (doi:10.1038/onc.2010.626)

Sfakianos GP \& Havrilesky LJ 2011 A review of costeffectiveness studies in ovarian cancer. Cancer Control 18 59-64.

Smith DM, Patel S, Raffoul F, Haller E, Mills GB \& Nanjundan M 2010 Arsenic trioxide induces a beclin1-independent autophagic pathway via modulation of SnoN/SkiL expression in ovarian carcinoma cells. Cell Death and Differentiation 17 1867-1881. (doi:10.1038/cdd.2010.53)

Uddin S, Ahmed M, Hussain A, Assad L, Al-Dayel F, Bavi P, Al-Kuraya KS \& Munkarah A 2010 Cyclooxygenase-2 inhibition inhibits PI3K/AKT kinase activity in epithelial ovarian cancer. International Journal of Cancer 126 382-394. (doi:10.1002/ijc.24757)

Wei Y, Kadia T, Tong W, Zhang M, Jia Y, Yang H, Hu Y, Tambaro FP, Viallet J, O'Brien S et al. 2010 The combination of a histone deacetylase inhibitor with the Bcl-2 homology domain-3 mimetic GX15-070 has synergistic antileukemia activity by activating both apoptosis and autophagy. Clinical Cancer Research 16 3923-3932. (doi:10.1158/1078-0432.CCR-10-0032)

Wirawan E, Lippens S, Vanden Berghe T, Romagnoli A, Fimia GM, Piacentini M \& Vandenabeele P 2012 Beclin1: a role in membrane dynamics and beyond. Autophagy 8 6-17. (doi:10.4161/auto.8.1.16645)

Zhang N, Wu ZM, McGowan E, Shi J, Hong ZB, Ding CW, Xia P \& Di W 2009 Arsenic trioxide and cisplatin synergism increase cytotoxicity in human ovarian cancer cells: therapeutic potential for ovarian cancer. Cancer Science 100 2459-2464. (doi:10.1111/j.1349-7006.2009. 01340.x)

Zhou F, Yang Y \& Xing D 2011 Bcl-2 and Bcl-xL play important roles in the crosstalk between autophagy and apoptosis. FEBS Journal 278 403-413. (doi:10.1111/ j.1742-4658.2010.07965.x)

Received in final form 17 August 2012 Accepted 21 August 2012 Made available online as an Accepted Preprint 23 August 2012 